Linguistique, littérature, didactique

151-152 | 2011

Anthropologies de la littérature

\title{
«Faire le Prussien ». Lecture ethnocritique de Saint-Antoine de Maupassant
}

\section{Véronique Cnockaert}

\section{(2) OpenEdition}

\section{Journals}

\section{Édition électronique}

URL : http://journals.openedition.org/pratiques/1836

DOI : $10.4000 /$ pratiques. 1836

ISSN : 2425-2042

\section{Éditeur}

Centre de recherche sur les médiations (CREM)

\section{Édition imprimée}

Date de publication : 15 décembre 2011

Pagination : 155-168

\section{Référence électronique}

Véronique Cnockaert, « «Faire le Prussien ». Lecture ethnocritique de Saint-Antoine de Maupassant », Pratiques [En ligne], 151-152 | 2011, mis en ligne le 13 juin 2014, consulté le 02 mai 2019. URL : http:// journals.openedition.org/pratiques/1836 ; DOI : 10.4000/pratiques.1836

(c) Tous droits réservés 


\title{
« Faire le Prussien ». Lecture ethnocritique de Saint-Antoine de Maupassant
}

\section{Véronique Cnockaert}

\author{
Université du Québec à Montréal, LEAL-FIGURA
}

Dans 1'édition de la Pléiade, Louis Forestier cherchant à définir la poétique de l'œuvre maupassantienne écrit au sujet de la campagne et de son personnel dans les contes et nouvelles :

Maupassant donne du paysan l'image qu'ont l'habitude de s'en faire ses lecteurs, gens des villes. Il façonne sa description sur un modèle littéraire qui l'empêche d'atteindre les fonds de l'âme campagnarde [...]. C'est qu'il ne s'intéresse pas à la paysannerie en tant que classe sociale, mais en tant que tempérament. Il étudie en elle une force de sentiments, une amoralité naturelle $[\ldots]^{(1)}$.

Le point de vue surplombant, qui serait celui d'une culture savante (ici le modèle littéraire) qu'accorde Forestier à Maupassant, s'il n'est pas faux, refuse au texte une partie de son ancrage culture ${ }^{(2)}$. Nous croyons en effet que dans ses descriptions du monde rural, Maupassant ne néglige pas la question sociale, mais plutôt démonte, souvent par le biais du comique, les systèmes de cohésion communautaire afin de mieux en saisir la nature et la teneur.

C'est justement la «présence d'un système culturel $^{(3)}$ » que nous voudrions étudier et plus précisément la mise en récit d'une pratique culturelle dans la courte nou-

(1) Louis Forestier, « Notices, notes et variantes » de Saint-Antoine (Contes et nouvelles de Guy de Maupassant, Paris, coll. «Bibliothèque de la Pléiade », t. I, 1974, pp. 772-779), p. XLI. Désormais, toutes nos références à l'œuvre renvoient à cette édition, suivies du numéro de la page entre parenthèses dans le texte.

(2) Il faudra attendre les travaux de Jean-Marie Privat (« Toine. Un géant chez Maupassant ", Actes du colloque Géants, dragons et animaux fantastiques en Europe (Ath), Tradition Wallonne, pp. 65-81; "Une chose malpropre et inutile. Approche ethnocritique de Boule de Suif », L'Autre en mémoire, Actes du Colloque international de Winnipeg (Canada), D. Laporte dir., Québec, Presses de 1'Université Laval, pp. 111-124) et ceux de Marie Scarpa ("Sauvage”, vous avez dit "sauvage" ? Lecture ethnocritique de La Mère Sauvage de Maupassant ", Littérature, n¹53, pp. 36-49) pour que cet aspect de l'œuvre soit étudié pour lui-même et non plus considéré comme une toile de fond.

(3) Jean-Marie Privat, Bovary-Charivari. Essai d'ethno-critique, «CNRS Littérature », CNRS EDITIONS, 2000 [1994], p. 18. 
velle Saint-Antoine, afin de révéler comment le populaire investit ce texte. Notre hypothèse générale repose sur l'idée qu'on peut mettre à jour un jeu de correspondances structurelles entre grammaire rituelle et efficacité narrative.

Il s'agit ainsi de montrer comment les us et coutumes qui parsèment le texte non seulement l'enrichissent, mais le structurent significativement. En effet, nous croyons que le détour par la tradition ne sert pas uniquement l'effet de réel et ne contribue pas simplement à teindre le récit d'une couleur locale. Nous pensons que les formes rituelles qui entourent les pratiques culturelles décelables dans le texte façonnent celui-ci fortement. Le « ré-ancrage culturel» que nous voudrions opérer devrait nous permettre de démontrer que le rite tel que « textualisé » par l'auteur en tant qu'espace privilégié de rassemblement social, se transforme d'une part en système de résistance partagé par la communauté et en tactique de guerre, d'autre part en partage ambivalent et en générosité équivoque. Dans cette histoire d'un village normand occupé par les troupes prussiennes, le rite, celui du gavage du cochon, semble bien l'arme privilégiée des dominés et l'angle mort que rencontre le dominant. Plus globalement, nous verrons que la réappropriation du scénario rituel par sa théâtralisation dysphorique permet à Maupassant de révéler qu'en temps de guerre, la barbarie ne se loge pas que chez l'ennemi, mais aussi là où l'on répugne à la trouver, c'està-dire en nous-mêmes.

La nouvelle Saint-Antoine est parue dans le journal Gil Blas en 1883, puis a été recueillie la même année dans Les Contes de la bécasse. Elle met en scène un paysan, prénommé Antoine qui, pour mieux prouver son opposition à l'occupation prussienne, engraisse le soldat prussien qui loge chez lui, le considérant comme un cochon. Cette cohabitation se termine le jour où, suite à une lutte entre les deux hommes, Antoine assassine le soldat. Par peur des représailles, le paysan cache son crime, mais alors que jusque-là Antoine faisait figure de résistant, sa crainte de la mort le transforme en ennemi de la nation puisqu'il laissera un innocent, et pas n'importe lequel, « un vieux gendarme en retraite, qui tenait une auberge dans le village voisin » (779) se faire fusiller à sa place. Notons que cette thématique de la vengeance des plus faibles, les paysans normands, contre le plus fort, l'occupant prussien, apparaît dans d'autres nouvelles, notamment Le Père Milon et La Mère sauvage.

Dans Saint-Antoine, le rapport inversé entre le comique du début et le tragique de la fin intensifie l'effet dramatique comme le laisse entendre Forestier dans son introduction qui souligne le détournement grotesque du motif du saint et de son cochon:

D'une certaine façon, à la fin du conte, Antoine est [...] à la recherche - qu'il sait inutile - de «son cochon ». Ces pages reposent donc sur une tradition populaire, détournée de manière grotesque et propre à rentrer dans ce qui était le dessein initial des Soirées de Médan : peindre «l'invasion comique». Pourtant, les toutes dernières lignes donnent une autre portée au conte, celle de tous ceux écrits sur ce thème par Maupassant : l'horreur, l'injustice et la dérisoire ironie des guerres ${ }^{(4)}$.

Le grotesque dont parle le critique se loge, on l'aura compris, non pas dans la transformation d'Antoine en saint Antoine, mais dans l'animalisation du Prussien. L'emploi que Forestier fait du mot grotesque renvoie à la définition de "réalisme grotesque » tel que défini par Bakhtine. Rappelons que ce « réalisme grotesque » se distingue par l'insertion dans le texte d'une imagerie ou de situations carnavales-

(4) Louis Forestier, «Notices, notes et variantes» de Saint-Antoine, op. cit., p. 1522. 
ques et par la présence d'éléments appartenant au comique populaire. Ainsi n'est-il pas faux d'envisager que la cochonnisation du Prussien et les rires que celle-ci provoque au sein du village font clairement référence à une culture festive, populaire et carnavalesque, le cochon étant dans nos sociétés un symbole de Carnaval.

Nous croyons que l'animalisation du soldat qu'esthétise le motif de « saint Antoine et son cochon » va cependant bien au-delà du pur effet comique. Il est clair que Maupassant ne cherche pas à transformer le temps de guerre en temps de carnaval - l'horreur des combats est encore trop vivace dans les mémoires pour qu' on puisse en rire. Il semblerait plutôt que l'écrivain, conscient que ces deux moments sont tous deux des temps de crise et de mise à l'envers du monde, les superpose. Il emprunte les extravagances de l'un pour décrire les absurdités de l'autre. Ce qui lie assurément guerre et carnaval, c'est que les catégories (sociale, morale, biologique) perdent en stabilité. En effet, les habitués du carnaval et les spécialistes de la question savent que c'est une période de liesse, de mascarades, d'injures autorisées, d'inversion des rôles (les dominants prennent la place des dominés et vice versa, les hommes deviennent des femmes et les femmes des hommes, beaucoup déambulent dans les rues grimés en animal), bref, c'est un moment durant lequel les hiérarchies sont mises à mal. À certains égards, il en va ainsi de la guerre : on n'est plus maître chez soi, les codes changent, les valeurs morales sont reconsidérées, ce qui autorise l'homme à faire preuve d'une barbarie qui le rapproche, on ne le sait que trop, de la bête. Les actes de guerre et les gestes carnavalesques obéissent ainsi d'une manière différente à une logique de renversement. Ce rapport d'équivalence permet de dire qu'en transformant symboliquement le soldat en cochon, Antoine, bien qu'il résiste à l'occupation, ne pose cependant pas un geste subversif; le paysan répond à l'inversion qu'impose le nouvel ordre établi, par une autre. De fait, à l'intérieur de cette dynamique de retournement, le dominé reprend sa place de dominant. On remarquera, cependant, que chacun des deux pôles de l'opposition, ici le paysan et le soldat, ne représente pas une valeur unique ou figée, l'un et l'autre sont, " simultanément ou successivement, positivement ou négativement marqués ${ }^{(5)} »$. Cette dialectique permet à Maupassant d'éviter les écueils du tout blanc ou du tout noir.

\section{La cochonnisation du Prussien}

Mais «pourquoi le Prussien en cochon»? Les discours médico-anthropologiques sur la supériorité ou l'infériorité des races qui fleurissent durant la seconde moitié $\mathrm{du} \mathrm{XIX}^{\mathrm{e}}$ siècle proposent une première réponse. Selon 1'historien Guy Bechtel qui étudie, dans son livre Délires racistes et savants fous, les théories pseudo-scientifiques de ce « racisme biologique » et ses dérives multiples, note que bien avant l'invasion prussienne, « [1]'Allemand, [...] constitue [déjà] la race la plus injuriée, la plus maltraitée d'Europe ${ }^{(6)} »$. Après la guerre de 1870 , il va sans dire que les discours haineux iront en s'amplifiant, les travaux du docteur Edgar Bérillon, professeur à l'École de psychologie de Paris, qui n'a de cesse de prouver l'infériorité physiologique de l'Allemand, en sont l'exemple le plus probant. Un parcours, même rapide, à travers ces différentes thèses, révèle une constante dans la description de

(5) Comme le remarque Marie Scarpa au sujet des oppositions et renversements qu'elle a répertoriés dans le roman Le Ventre de Paris de Zola (Le Carnaval des Halles. Une ethnocritique du Ventre de Paris d'Émile Zola, CNRS éditions, Paris, 2000, p. 214).

(6) Guy Bechtel, Délires racistes et savants fous, Paris, Plon, 2002, p. 129. 
l'Allemand, qui fonde, semble-t-il, sa spécificité. Ce dernier posséderait un appétit hors du commun qui le porterait à manger de manière avide et sans raffinement. Louis-Auguste Blanqui, le 12 septembre 1870, dans article patriotique « La patrie en danger ", explique cette gloutonnerie par une raison anatomique, selon lui, le Germain aurait « un mètre de tripes de plus que le Français » ${ }^{(7)}$. On le sait, la proximité entre l'homme et l'animal passe généralement - les plaisanteries et les proverbes en font foi - par des goûts alimentaires identiques. ${ }^{(8)} \gg$ Ces caractéristiques, goinfrerie native associée à une grossièreté dans les manières et les goûts, plus que de le rapprocher, assimilent l'Allemand au cochon.

Avant qu'Antoine rencontre le Prussien, on ne s'étonnera donc pas qu'il parle des soldats ennemis en termes de nourriture et qu'il se «prom[et] de manger une armée » (772), «Faudra bien que j'en mange » (ibid.) insiste-t-il sur un ton hâbleur. On remarque ensuite que la première description du Prussien installe d'emblée une parenté physiologique entre cet homme et l'animal, et ce même avant que le paysan n'est l'idée de faire de lui « son cochon »: "C'était un gros garçon à la chair grasse et blanche, aux yeux bleus, au poil blond, barbu jusqu'aux pommettes, qui semblait idiot, timide et bon enfant. ${ }^{9)} \gg(773)$ On voit que la description s'appuie donc clairement sur les valences sémiques du cochon (chair grasse, poil blond). Si les discours modélisants permettent de comprendre l'homologie descriptive entre le Prussien et l'animal porcin, ils ne permettent cependant pas d'expliquer pourquoi toute la communauté adhère si rapidement à la plaisanterie d'Antoine et l'encourage à poursuivre cette comédie ; plus encore, y participe.

Le rite, comme système culturel, assure la cohésion sociale, il permet aussi de dominer les événements plutôt que d'être dominé par eux. Nous verrons que la cochonnisation du Prussien offre effectivement à la communauté, condamnée à supporter la présence de l'ennemi jusque dans l'intimité des familles, une manière d'intégrer (pour mieux s'en séparer) celui qui représente, on en conviendra, l'étranger absolu. Soulignons tout de suite, comme l'observe l'ethnologue Yvonne Verdier, que dans nos campagnes,

[m]anger du lard, et du lard bien gras, est aussi une preuve physique dont la portée est toute morale. Que de tous jeunes enfants « mangent du lard gras de bon cœur» témoigne de leur force [...]. Qu'un homme, en revanche, soit trop faible d'estomac pour en consommer est $[\ldots]$ signe d'autres faiblesses $[\ldots] .^{(10)}$

C'est donc sur le dos du Prussien transformé en cochon que la communauté entend puiser les forces de sa résistance, et ce de manière inattendue, puisque festive. Tout aussi inattendue la façon qu'a Antoine de prouver «à son monde» (773) qui s'attend «à le voir écharper le Prussien» (ibid.) qu'il est un homme de parole. Dans ce village

(7) Auguste Blanqui, «La patrie en danger», 12 septembre 1870. Cité par G. Bechtel, ibid., p. 143.

(8) Colette Mechin, Bêtes à manger. Usages alimentaires des Français, Presses universitaires de Nancy, Nancy, 1992, 270 p., ici p. 18. On retrouve ce rapprochement par la manière de manger entre le Prussien et le cochon dans Boule de suif: "Ces gens-là, ça ne fait que manger des pommes de terre et du cochon, et puis du cochon et des pommes de terre. Et il ne faut pas croire qu'ils sont propres. - Oh non! Ils ordurent partout. » (Maupassant, Boule de suif). Sur le prétendu manque de tenue des Prussiens, voir Jean-Marie Privat, "Une autre chose mal propre et inutile". Approche ethnocritique de Boule de suif ", Actes du Colloque international de Winnipeg (Canada), D. Laporte dir., Québec, Presses de 1'Université Laval, 2006, pp. 111-124, note 15.

(9) On retrouve cette homologie descriptive Prussien/cochon dans Boule de suif, ainsi que dans $L a$ Débâcle d'Émile Zola.

(10) Yvonne Verdier, Façons de dire, façons de faire. La laveuse, la coutière, la cuisinière, Paris, Gallimard, «nrf», 1979, p. 24. 
du pays de Caux où l'on sait, et le narrateur aussi, que tout cochon est voué un jour à être sacrifié, Antoine, en cochonnisant le soldat, le convertit en bête à tuer.

Historiens et ethnologues s'entendent pour souligner la proximité historique de 1'homme et du cochon. Ainsi, Claudine Fabre-Vassas qui a consacré une importante étude aux rapprochements problématiques entre le cochon, le chrétien et le juif à travers les âges, rappelle la similitude anatomique entre l'homme et la bête : ouverte, elle est « notre miroir, elle révèle une commune origine décelable dans les viscère, le sang et certain os ${ }^{(11)} »$. Philippe Walter explique, quant à lui, dans son introduction aux Actes du colloque Mythologie du porc, que

le cochon est un miroir déformant de l'humanité ; plus qu'aucun autre animal, il semble renvoyer l'homme à sa propre nature ; il interroge l'homme sur son propre comportement ou sur ses excès. À vrai dire, le porc est un animal paradoxal. Il nourrit les hommes depuis des temps immémoriaux et subit néanmoins un statut extrême : tantôt considéré comme le meilleur ami du genre humain, tantôt frappé de graves interdits philosophiques et religieux, le porc semble toucher aux valeurs fondatrices de nombreuses civilisations. $^{(12)}$

Cette capacité de mirer notre humanité éclaire le fait que cet animal, ainsi que le note Marie Scarpa, " offre sans doute dans nos cultures, le spectre le plus large de connotations, [...] : ce qui lui permet d'entrer dans un réseau métaphorique particulièrement dense et d'avoir une place non commune dans les pratiques culturelles comme dans les discours qui le sollicitent ${ }^{(13)} »$.

En effet, les modalités qui entourent cet animal rendent compte de la nature antinomique que nos sociétés lui attribuent. Il suffit d'étudier la manière dont on l'élève dans les campagnes pour s'en convaincre. Bien qu'il soit l'un des animaux les plus rejetés, il est un de ceux dont on prend, collectivement, le plus soin. C'est que « faire le cochon », c'est-à-dire « le tuer, le découper et distribuer ces parts dans l'espace social $^{(14)}$ », c'est « d'abord l'élever ${ }^{(15)}$ » comme le remarque Claudine Fabre-Vassas. Le terme " élever» n'est pas trop fort, car après avoir acheté un pourceau, la famille, et notamment la femme, s'en occupe « de façon maternelle ${ }^{(16)} »$. Elle va le nourrir comme un enfant, et entretenir avec lui un rapport d'exclusivité. Cette prise en considération de l'animal rend son statut hautement problématique. En élevant le petit cochon comme un membre de la famille, en le rapprochant symboliquement de la nature humaine (dans certains villages, on va jusqu'à ressentir le besoin de le baptiser du titre de «Monsieur », plus rares sont les villages où on lui donne un nom propre), on « risque, note Fabre-Vassas, de faire surgir le spectre d'un insupportable cannibalisme ${ }^{(17)} »$. C'est pourquoi, l'infâme réputation qui précède l'animal sert aussi de garde-fou et de mise à distance permanente afin que l'on n'oublie jamais, malgré les nombreux soins dont il bénéficie, que l'animal est vil et féroce et qu'il convient de le tuer. Le tuer, c'est aussi d'une certaine manière réaffirmer son statut de bête et en finir avec cette insupportable proximité. Nous verrons que la tentation du canniba-

(11) Claudine Fabre-Vassas, La Bête singulière. Les juifs, les chrétiens et le cochon, Paris, Gallimard, «nrf», 1994, p. 288.

(12) Philippe Walter, (dir.), «Le porc, animal mythique», dans Mythologie du Porc. Actes du colloque de Saint-Antoine l'Abbaye (Isère) 4 et 5 avril 1998, Grenoble, Jérôme Million, p. 7.

(13) Marie Scarpa, Le Carnaval des Halles, op. cit., p. 189.

(14) C. Fabre-Vassas, op. cit., p. 9.

(15) Ibid.

(16) Y. Verdier, Façons de dire, façon de faire, op. cit., p. 25

(17) C. Fabre-Vassas, op. cit., p. 11. 
lisme n'est pas étrangère au texte de Maupassant, elle apparaît une première fois sous forme de farce alors que les villageois s'amusent à dépecer oralement le Prussien («-J'te l'vends, Césaire, trois pistoles. / - Je l'prends, Antoine, et je t'invite à manger du boudin. / - Mé, c'que j'veux, c'est d'ses pieds. » (774)), et elle réapparaît à la fin du récit de manière détournée comme un refoulé, nous y reviendrons. On ne peut cependant pas s'étonner de cet horizon, étant donné que la représentation du Prussien en cochon fait de lui une «bête à manger».

Mais revenons à la vie du cochon dans la famille. Parce qu'il est considéré comme un enfant, voir un nourrisson, le régime qu'il supporte durant les premiers temps de son gavage est essentiellement constitué de soupe ou de purée, ainsi fait-on fi de sa forte dentition et de son statut d'animal dangereux ${ }^{(18)}$. Sa violence lui sera restituée lorsque viendra le temps de le tuer, de telle sorte qu'aux yeux de ceux qui l'ont élevé et gâté, le crime soit légitimé et facilité ${ }^{(19)}$.

Le gavage du cochon s'il est une affaire de famille permet aussi de tisser ou de réaffirmer des liens entre celle-ci et les membres de la communauté, puisque les voisins qui contribuent au gavage auront droit, lors de la tuée, à une part de la bête. On appelle cette action " "porter" au cochon ${ }^{(20)}$ ". Un véritable espace de sociabilité s'organise ainsi autour de l'animal. En effet, on dit de ceux qui participent à cette économie de voisinage basée sur l'élevage et sur le partage équitable de la bête qu'ils sont « en relation de cochon ${ }^{(21)}$ ».

Si l'on revient à notre texte, il est facile de s'apercevoir que l'arrivée du Prussien dans la cuisine d'Antoine renvoie à la première étape de gavage du petit cochon. Le fermier, après avoir scruté l'homme avec l'œil du connaisseur qui ne se trompe pas sur la qualité des bêtes - «Le Normand malin le pénétra tout de suite, et, rassuré, lui fit signe de s'asseoir » (773) -, et s'être assuré que le Prussien ne saisissait pas un mot de français, lui offre d'emblée, non pas une assiette, mais trois assiettes de soupe. La parole accompagne le geste de cette entrée en cochonnisation du soldat puisque le paysan ne craint pas de dire, en lui mettant la pitance « sous le nez » (ibid.) :

«Tiens, avale ça, gros cochon. »/ Le soldat répondit « Ya » et se mit à manger goûlument [...]. Quand le Prussien eut englouti son assiettée, Saint-Antoine lui en servit une autre qu'il fit disparaître également; mais il recula devant la troisième, que le fermier voulait lui faire manger de force, en répétant : "Allons fous-toi ça dans le ventre. T'engraisseras ou tu diras pourquoi, va, mon cochon!» (ibid.)

Nous remarquons que le processus d'animalisation du soldat est double. D'une part, il possède les manières et la physionomie du cochon et d'autre part, il est dépossédé de sa parole et même de la raison (plusieurs fois son air "stupide » est souligné dans le texte). Ajoutons que le lecteur n'aura pas accès au nom du soldat, toujours nommé par le narrateur et les personnages «l'étranger», «le Prussien », « le soldat» ou «mon cochon ». Alors que l'absence de nom éloigne le cochon de la troublante proximité qu'il entretient avec les hommes, en revanche la disparition de nom dans le cas du Prussien rapproche l'homme de cet animal. Ainsi pourvu et dépourvu, le

(18) Ibid., p. 59.

(19) Ainsi que le rappelle Y. Verdier : «On s'attache à lui et on a mal au cœur le jour où il faut le tuer. Aussi un beau matin, on décide que l'animal est devenu "méchant", comme pour alléger le crime ». op. cit., p. 25.

(20) Ibid., p. 25.

(21) Ibid., p. 27. 
Prussien n'est plus l'étranger venu d'ailleurs, mais plutôt celui qui est étranger à la culture locale, et plus encore à la civilisation, en tant qu' homme devenu " cochon ». L'animalisation du soldat invite et autorise Antoine et les villageois à le domestiquer. Or dans nos sociétés, la domestication du cochon passe par son gavage en vue de la tuée. Nous ne serons donc pas surpris que le récit progresse en respectant, dans l'ordre, les étapes du rite : 1 . le gavage par un individu puis par la communauté ; 2. l'ensauvagement (il faut rendre au cochon sa méchanceté native) ; 3 . la tuée.

\section{Le gavage}

On remarque en effet que soldat est tout d'abord gavé par Antoine, avec lequel il entretient un rapport d'exclusivité et auquel il va s'attacher comme l'animal à son maître :

Ils étaient devenus, d'ailleurs, les meilleurs amis du monde; et quand le vieux allait à ses affaires dans les environs, le Prussien l'accompagnait de lui-même pour le seul plaisir d'être avec lui. (775)

Ensuite, le gavage est pris en charge par les villageois :

Dès lors, le père Antoine ne sortit plus sans son Prussien. [...] / Ils s'en allaient tous les jours après-midi, bras dessus bras dessous avec son Allemand [...]. / Et il avait pris l'habitude de faire offrir à manger à son cochon partout où il entrait avec lui. C'était là le grand plaisir, le grand divertissement de tous les jours [...]. Et on offrait à l'homme du bain et du beurre, des pommes de terre et du fricot froid, de l'andouille qui faisait dire : "De la vôtre, et du choix" ${ }^{(22)} »(774)$. [...] «Et chaque jour c'était une fête de nourrir l'animal. Tout le pays accourait là comme on va, le dimanche à la grand'messe. » (775).

Les références à la fête et à la messe signalent que l'action de nourrir le Prussien se transforme rapidement en opération de rassemblement communautaire ${ }^{(23)}$. Seuls la transformation symbolique du soldat en cochon et donc en bête à tuer, et la théâtralisation de son gavage, expliquent un tel consensus social, sinon comment comprendre que les villageois donnent aussi joyeusement et généreusement à manger à celui qui est d'abord l'ennemi? G. Bataille, dans La Part maudite, explique bien cette position équivoque de la victime : bien qu' « abandonnée à la violence ${ }^{(24)}$ », «[r]ien n'est plus frappant que les soins dont on l'entoure. ${ }^{(25)} \gg$ De fait, l'abondance et la qualité de la nourriture, sa richesse en matière grasse, prouvent qu'il ne s'agit pas tant de nourrir l'homme que de l'engraisser comme un cochon, ce qui ne manque pas d'advenir :

Le soldat, stupide et doux, mangeait par politesse, enchanté de toutes ces attentions, se rendait malade pour ne pas refuser ; et il engraissait vraiment, serré maintenant dans son uniforme, ce qui ravissait Saint-antoine et lui faisait répéter : « Tu sais, mon cochon, faudra te faire faire une autre cage. » (775)

(22) Cette dernière remarque du fermier renvoie de manière humoristique au cannibalisme notoire du cochon sur laquelle nous aurons à revenir, car cette question n'est pas sans incidence sur la dimension symbolique du texte que l'on devrait être en mesure de préciser au terme de cette analyse.

(23) Dans son ouvrage, Marie Scarpa rappelle que le cochon avant tout « est un aliment festif ». (op. cit., p. 193 et suiv.).

(24) Georges Bataille, La Part maudite dans Euvres complètes, «nrf », Gallimard, t. VII, p. 64.

(25) Ibid., pp. 64-65. 


\section{L'ensauvagement}

Cette plaisanterie sur la taille de l'uniforme, outre qu'elle soit un outrage direct fait à l'uniforme prussien (et à la nation qu'il symbolise) en installant un rapport d'équivalence entre l'habit du soldat et l'habitat du cochon, correspond à la fin de la première phase de développement du jeune cochon quand il arrive à maturité. En effet, l'indication temporelle qui suit cet épisode, évoque l'« hiver de $1870 »$ (ibid.). Or, le sacrifice du cochon a généralement lieu l'hiver pour des raisons économiques d'une part, car « le lard baisse dans le saloir ${ }^{(26)}{ }$ et d'autre part, pour des raisons festives (Noël, Jour de l'an, fêtes de famille). Dans tous les cas, « il faut opérer avant les chaleurs » dit-on, pour éviter que le lard ne devienne rance. Il est préférable aussi, selon les villageois auprès desquels a enquêté Yvonne Verdier, de tuer et de commencer à cuisiner la bête un jour de « lune vieille », c'est-à-dire une nuit sans lune, le lard et le boudin n'en seront que meilleurs ${ }^{(27)}$. Cette règle qui appartient au domaine du magico-religieux n'aurait que bien peu d'importance à nos yeux si le texte ne précisait que la mort du soldat avait, elle aussi, lieu lors d'une «nuit sans lune » (776). À ces raisons calendaires qui précisent le temps du sacrifice, se superpose une raison physiologique, celle qui veut que lorsque le cochon atteint l'âge adulte, il devient dangereux. Et si tel n'était pas le cas, on travaille à le rendre méchant par un changement de régime et/ou par la claustration. Cet « ensauvagement» du cochon s'avère nécessaire afin que le sacrifice soit supportable à ceux qui se sont attachés à l'animal. En le rendant à sa sauvagerie, les liens privilégiés qu'entretenait avec lui son propriétaire, ainsi que tous ceux qui étaient « en relation de cochon » (ici le village au complet visiblement) se distendent, par conséquent la tuée est, non seulement possible, mais acceptée.

Ces chronologies et ces pratiques sont lisibles dans le texte de Maupassant. Suite à l'indice temporel («le terrible hiver de $1870 »)$, nous remarquons que le Prussien jusqu' ici bon enfant, prêt à se « rend[re] malade pour ne pas refuser » (ibid.) la nourriture qui lui est offerte, devient méfiant : « quand on riait trop fort il roulait des yeux inquiets qui, parfois, s'allumaient d'une flamme de colère » (ibid.). Cette transformation ne vient pas seule, arrive le moment où pour la première fois depuis le début du gavage, le soldat repus, refuse « d'avaler un morceau de plus » (ibid.). Cette rebuffade est perçue par Antoine comme un acte de désobéissance. Pour remédier à cette situation nouvelle et pour rasseoir son autorité, le fermier impose au soldat un changement de régime : "Puisque tu n'veux pas manger, tu vas boire, nom de Dieu ! » (ibid.) et Antoine de le forcer à avaler de l'eau-de-vie. Ce changement de régime qui met clairement fin à la période de gavage proprement dite est accompagné d'une décision d'Antoine qui entame la dimension collective du rite, lorsque ce dernier choisit de suivre le soldat sur le terrain de la boisson dans un vrai duel d'alcooliques. Par ce geste singulier et personnel, Antoine quitte le terrain rituel, espace partagé par toute la communauté, pour celui de la « lutte» (776), de la «bataille» (ibid.), de la «revanche» (ibid.) d'un homme avec un autre homme. La suite des événements confirme ce passage d'un ordre collectif à un ordre personnel puisque désormais les seuls acteurs, au sens fort du terme, du drame seront Antoine et le Prussien. Sur le plan symbolique, ce déplacement n'est pas sans conséquence, le duel marque le passage d'un régime du sacrifice pour celui de l'assassinat criminel.

De fait, l'ivresse partagée ne fait pas qu'exclure les deux individus du cercle de la

(26) Y. Verdier, op. cit., p. 26.

(27) Ibid. 
communauté, elle les installe également dans un rapport d'équivalence : «Ils n'en pouvaient ni l'un ni l'autre quand le litre fut séché. Mais aucun des deux n'était vaincu. Ils s'en allaient manche à manche, voilà tout. Faudrait recommencer le lendemain ! » (ibid.) Or, l'histoire nous révèle que le duel prochain est imminent. À peine sortis de la ferme des Haules s'engage, à l'initiative d'Antoine, un jeu corporel entre les deux hommes qui se mue rapidement en bagarre.

[...] Saint-Antoine, mécontent de n'avoir pas triomphé, s'amusait à pousser l'épaule de son cochon pour le faire culbuter dans le fossé. L'autre évitait les attaques par des retraites ; et, chaque fois, il prononçait quelques mots allemands sur un ton irrité qui faisait rire aux éclats le paysan. À la fin, le Prussien se fâcha; et juste au moment où Antoine lui lançait une nouvelle bourrade, il répondit par un coup de poing terrible qui fit chanceler le colosse. (ibid.)

Cet épisode redit que la frontière entre dominant et dominé est abolie et qu'il s'agit pour l'un comme pour l'autre de reconquérir la place du plus fort qu'ils ont occupé auparavant tour à tour : le Prussien en tant que conquérant, le fermier en tant que propriétaire du cochon. Certains indices explicitent clairement ce rapport d'équivalence, en effet, bien qu' Antoine persiste à considérer le soldat comme " son cochon » (ibid.), on remarque que le Prussien en colère a recouvré la parole : rendu à sa méchanceté, l'homme est aussi rendu à sa culture. Il redevient l' « étranger » que l'on peut tuer.

\section{La tuée}

Il n'est donc pas surprenant qu'Antoine pris tout entier dans le jeu mimétique du rite-tout comme la logique textuelle du récit-, assomme préalablement le Prussien, comme il est coutume de faire avec le cochon, avant de le « saigner». Effectivement, après avoir laissé le soldat qu'il croyait mort dans un tas de fumier, ce n'est que le lendemain, alerté par son chien, qu'Antoine tue de plusieurs coups de fourche le Prussien «encore hébété par l'ivresse, étourdi par le coup, épuisé par sa blessure » (778) :

Le soldat se renversa sur le dos en poussant un long soupir de mort, tandis que le vieux paysans, retirant son arme des plaies, la replongeait coup sur coup dans le ventre, dans l'estomac, dans la gorge, frappant comme un forcené, trouant de la tête aux pieds le corps palpitant dont le sang fuyait par gros bouillons. (779)

Tourmenté à l'idée qu'on retrouve le cadavre, le paysan l' « enseveli[t] » (ibid.) dans la terre sous le fumier. En abandonnant le cadavre à la décomposition, Antoine impose un outrage extrême au corps de l'ennemi qui « ainsi dissous dans la confusion, [est] renvoyé au chaos [pour le punir de celui qu'il a créé], à une entière inhumanité ${ }^{(28)} »$. Antoine, on le sait, ne se glorifiera pas de ce crime, bien au contraire, il laissera un innocent se faire condamné et fusillé à sa place. Mais avant d'en arriver à la fin du récit, interrogeons celle du soldat à la lumière des pratiques qui entourent le sacrifice du cochon.

On l'a dit plus tôt, « faire le cochon », c'est, après l'avoir élevé, engraissé et tué, le découper et distribuer ces parts avec ceux et celles qui étaient en « relation de co-

(28) Jacques Sémelin, Purifier et détruire. Usages politiques des massacres et génocides, Paris, « La couleur des idées », Seuil, 2005, p. 356. 
chon ». En quittant la sphère publique du rite pour la sphère privée du duel - ce qui par ricochet évacue la dimension sacrificielle du crime -, le récit semble éloigner «le spectre du cannibalisme ${ }^{(29)}$ » qui hante la narration depuis le début, vu que le Prussien en tant que bête à manger n'est plus. Et pourtant, le lieu d'ensevelissement du cadavre est de ce point de vue lourd d'inquiétudes. On sait que le fumier qui est un espace de transformation, est un lieu doublement connoté : négativement parce qu'il est le lieu de récupération des déjections animales, et positivement parce que sous l'action de la fermentation, le pourri se mue en engrais, en source de vie. C'est pourquoi, en mêlant le cadavre du Prussien aux excréments, Antoine s'assure que la dépouille après décomposition se transforme en fertilisant, de telle sorte qu'il tient contre toute attente sa promesse de répartition du corps du Prussien. Ainsi, bien que sur le plan narratif et anthropologique le sacrifice du soldat n'a pas eu lieu, sa mort étant le résultat d'un meurtre, il n'en reste pas moins que sur le plan symbolique, le corps de la victime, "en tant que bête à manger» est rendu à la communauté. En effet, après avoir été engraissé par la collectivité, le soldat décomposé va engraisser celle-ci par capillarité.

Toute la dimension sacrée de la violence faite au corps du soldat depuis le premier gavage jusqu'au coup de fourche retentit dans ses agapes inopinées comme un écho tardif. Cela dit, rien dans le geste d'Antoine et dans le partage différé du corps de la victime ne permet de dire qu'il y a efficacité du rite. Bien au contraire, alors que le rite est instrument de cohésion sociale, lieu de communion et de résolution des conflits, la mort du gendarme, dit que le désordre et la violence créés par la venue des Prussiens dans le village est encore plus grande après la mort du soldat qu'avant, puisque désormais on laisse par couardise mourir un compatriote (et pas n'importe lequel, étant donné qu'il s'agit d'un représentant de la nation).

$\mathrm{Si}$, au départ, la nouvelle ravive l'idée que la guerre est un moment de forte socialisation ${ }^{(30)}$, elle révèle surtout qu'il ne faut pas confondre sursaut collectif et solidarité : avoir un ennemi commun n'invite pas forcément à la fraternité.

Alors qu'on aurait pu croire que la cochonnisation du Prussien servirait dans ce contexte de guerre d'outil symbolique pour corriger les spoliations et rendre aux dominés leur place de dominants et leur dignité, la fin du récit dont l'alternative est d'un côté le cannibalisme et de l'autre, l'abandon d'un vieux gendarme innocent aux mains de l'adversaire, rappelle, qu'en cette année 1870, la patrie reste livrée à la barbarie, à toutes les barbaries, à celle de l'envahisseur et à celle des patriotes, et plus insupportable encore, à celle de ces derniers entre eux. En somme, l'idée sous-jacente du texte est que dans l'avilissement de l'autre (même si cet autre est l'ennemi), l'homme s'avilit lui-même, ce qui fait de lui un animal, en l'occurrence un porc, qui, par ces actions, emmène la patrie dans la fange comme le sous-tend l'horizon «cannibalique » du texte. En effet, la réputation de bête féroce qui entoure le cochon doit au fait qu'il est non seulement capable de s'attaquer à la main qui le nourrit et de dévorer des enfants (selon Claudine Fabre-Vassas, l'animal aurait « donn[é] corps au croquemitaine $\left.^{(31)} \gg\right)$, mais aussi que la truie n'hésite pas à manger sa progéniture. La remarque que fait Antoine au soldat lorsqu'il lui donne de l'andouille à manger, " "De la vôtre, et du choix" " (774), renvoie directement au cannibalisme notoire de la bête. Si bien que la transmutation du soldat en engrais, qui convertit les villageois en cannibales, les transforme également, dans l'économie romanesque, en cochons.

(29) Claudine Fabre-Vassas, op. cit., p. 11.

(30) Comme le rappelle Roger Caillois dans L’homme et le sacré, Paris, Gallimard, « Idées », 1950.

(31) Claudine Fabre-Vassas, op. cit., p. 99. 
La morale de l'histoire pourrait être : « À force de "faire le cochon", on le devient ». Derrière la cochonnisation du Prussien, le récit qui ne fait preuve, on l'aura compris, d'aucune volonté édifiante, témoigne donc de l'ensauvagement des hommes par la guerre. La réélaboration rituelle du gavage qui structure le texte marque ainsi la distance du romancier face à l'Histoire, face à la guerre et face à la nation, elle exprime finement les ambiguïtés et les paradoxes qui traversent l'écrivain et façonnent le récit.

\section{Antoine en saint Antoine}

En effet, le texte va bien au-delà de « la dérisoire ironie des guerres ${ }^{(32)} »$ dont parle Louis Forestier dans sa présentation. Il semble même que la mise en récit du rite du gavage construise des « effets d'empathie ${ }^{(33)}$ » ambivalents. Effectivement, 1'acte de gaver qui joue dans le texte sur la double valence du plaisir et de son au-delà qui se loge dans la douleur, se transforme rapidement sous nos yeux en acte de torture. On a vu que le geste imposé par l'ennemi (nourrir le Prussien) a été transformé sous l'impulsion d'Antoine en rite et en acte de résistance, c'est donc un geste qui possède une valeur positive. C'est ce même geste cependant qui est à l'origine de la compassion $\mathrm{du}$ lecteur pour le soldat, car il possède également une valeur négative en tant qu'acte de persécution. Si la nature accorte du Prussien et ses prouesses de bonne volonté facilite le processus d'empathie du lecteur pour le personnage, celle-ci est également favorisée par la personnalité vigoureuse et le tempérament sanguin d'Antoine qui contraste avec le tempérament conciliant, voire bonasse, du Prussien.

Sans doute pouvait-on prévoir dès le début que le fermier ne serait pas le sauveur qu'il promettait d'être, et que le programme narratif annoncé par le titre devait être considéré de manière ironique. Antoine partage, il est vrai, peu de similitude avec le saint dont il porte le nom :

On l'appelait Saint-Antoine, parce qu'il se nommait Antoine, et aussi peut-être parce qu'il était bon vivant, joyeux, farceur, puissant mangeur et fort buveur, et vigoureux trousseur de servantes, bien qu'il eût plus de soixante ans.

C'était un grand paysan de Caux, haut en couleur, gros de poitrine et de ventre, et perché sur des longues jambes qui semblaient trop maigres pour l'ampleur du corps. (772)

Malgré le peu d'homologie entre le saint et le fermier, la référence au premier s'arc-boute d'emblée sur une tradition iconographique qui éveille dans l'imaginaire occidental la figure de l'ermite accompagné de son cochon. Ceci crée chez le lecteur de la nouvelle un horizon d'attente puissant, celui de la venue prochaine de l'animal. Conscient de cette efficacité narrative, Maupassant prend soin, dans la description du fermier, de le rapprocher immédiatement de son attribut principal (« puissant mangeur », " gros de poitrine et de ventre, perché sur des [...] jambes trop maigres pour l'ampleur du corps »). Le procédé métonymique évoque en sourdine qu'Antoine est habité par l'esprit du mal (esprit qui prit, on le sait dans la tradition, entre autres formes, celle d'un cochon). Ces détails textuels ont pour conséquence de diaboliser le paysan en le construisant en mauvais sujet et de désacraliser le saint dont il porte le nom comme un étendard. L'incipit, dont on connaît l'importance en régime roma-

(32) Louis Forestier, op. cit., p. 1522.

(33) Nous empruntons le syntagme à Marie Scarpa dans «Sauvage, vous avez dit "sauvage” ", op. cit. 
nesque, en insistant sur la physionomie porcine du paysan donne la préférence à la part sombre du saint et non pas à sa part lumineuse. Les caractéristiques de « farceur», de «trousseur » et de «buveur», qui inscrivent Antoine et le texte dans une logique d'inversion et une esthétique de carnaval plutôt que religieuse, redoublent ce processus de désacralisation que les qualificatifs dépréciatifs (" hâbleur ", « couard », " sournois ») perpétuent et achèvent. Ainsi, dès l'ouverture, la nouvelle prend ses distances face aux valeurs morales qu' incarne l'ermite pour insister sur les relations symboliques et ambivalentes que le personnage entretient avec le cochon. À l'intérieur de cette carnavalisation textuelle, il ne faut pas s'étonner que l'animal qui accompagne ici notre Saint-Antoine soit représenté sous les traits, comme on l'a déjà souligné, d' " un gros garçon à la chair grasse et blanche, aux yeux bleus, au poil blond, [...] qui sembl[e] timide et bon enfant » (773). Bien que nous sachions qu'il s'agit d'un soldat ennemi, bien que la description réunisse sous des caractéristiques communes l'homme et le cochon, rien dans celle-ci ne permet d'attribuer au personnage le caractère démoniaque qu'accorde la tradition religieuse à l'animal (au-delà des tentations de saint Antoine, pensons à l'épisode des «démons et [d]es cochons" dans l'Évangile de saint Matthieu ${ }^{(34)}$ ). Et plus nous avançons dans le texte et plus la narration nourrit notre empathie, le récit maupassantien ne cessant de souligner les sentiments d'amitié que le soldat développe pour le paysan. On aura compris que cette amitié à sens unique rend le geste meurtrier d'Antoine encore plus violent et plus brutal qu'il s'inscrit dans une relation interpersonnelle, même si l'épisode du duel rétablit une manière d'équilibre en insistant d'une part sur la férocité du Prussien et d'autre part, sur la bouffonnerie d'Antoine.

On remarque donc que le texte travaille habilement à placer le lecteur en position de solidarité affective avec le Prussien, comme les villageois avec leur cochon. Tout comme eux qui, de diverses manières, ensauvagent la bête pour la mettre à distance afin de supporter sa tuée, c'est presque avec soulagement que le lecteur qui sait la mort du soldat inévitable le voit renouer avec la violence. Dans ces circonstances, nous croyons pouvoir dire que d'une certaine manière le lecteur « fait lui aussi le cochon », car s'il ne nourrit pas le Prussien, il nourrit le personnage de son empathie. La prouesse de Maupassant est qu'en textualisant le rite, il permet au lecteur d'y participer en partie, cependant il ne l'invite pas à honorer la mort du Prussien, même si le geste meurtrier d'Antoine reflète les opinions exprimées en 1870. Au contraire, la narration multiplie les moyens pour empêcher le regard complice, afin de nous rappeler que la guerre, comme le carnaval d'ailleurs, réveille la mentalité sauvage qui sommeille en l'homme. C'est un moment de désordre complet où la cohésion sociale est suspendue, où les repères disparaissent. Mais autrement que la fête, la guerre entame l'efficacité des rites qui ne sont plus désormais garants d'un retour à l'ordre. Les rites, les sacrifices, partenaires essentielles des fêtes sont dans la nouvelle Saint-Antoine pervertis et vains, car nous sommes en guerre. C'est que la coutume, les fêtes, le rite « rappelle[nt] aux hommes l'ordre répétitif des choses ${ }^{(35)}$ », alors que la guerre interrompt comme un coup du destin cette rassurante répétition. Ce que Maupassant met ainsi en évidence « en croisant coutume et destin ${ }^{(36)}$ », pour repren-

(34) La Bible, «Évangile selon saint Matthieu », 8, 28. "Pour beaucoup de Normands, le nom de 1'ermite évoquait une quête gémissante et cocasse [mise en scène par le marionnettiste Legrain] : Messieurs les démons, laissez-moi donc!

Rendez-moi mon cochon, s'il vous plaît,

Voulez-vous me le rendre..

Cité par Louis Forestier, Notice, Notes et variantes dans Contes et nouvelles, op. cit., p. 1522.

(35) Y. Verdier, Coutume et destin, op. cit. p. 159.

(36) Ibid., p. 157. 
dre la formule d'Yvonne Verdier, c'est que le destin est plus fort que la coutume et que la guerre est plus forte que les hommes. La guerre ne promet aucune glorification de la patrie (désormais on l'assassine), et les bons et les méchants ne sont même pas des données objectives : Antoine n'a rien d'un saint et le Prussien rien d'un cochon. Aucune apologie de la coutume donc, au contraire pourrions-nous dire, ce que montre l'écrivain, c'est que sous couvert de rite, la barbarie fonctionne complètement, quoique déguisée. Cependant, si Maupassant a choisi ici le rite du gavage du cochon pour illustrer l'horreur de la guerre, c'est qu'il est d'une redoutable efficacité narrative. En effet, en considérant que le but ultime de la guerre est l'élimination ou l'assimilation complète de l'autre, le gavage et la tuée du cochon offrent un espace illustratif tout à fait original, mais surtout extrêmement opérant. C'est un fait que l'analyse ethnocritique permet d'ouvrir le texte sur des dimensions fondamentales du texte de Maupassant, celles de la conquête de l'autre et de son incorporation.

La métamorphose du Prussien en cochon est de ce point de vue significative. En devenant aux yeux des villageois un " cochon », le soldat, nous l'avons vu plus tôt, perd son identité d' « humain », dès lors il se mue aux yeux de la communauté en « animal sacrificiel », c'est-à-dire qu'il fait culturellement sens, et devient un signe assimilable par tous. Par ailleurs, cette mutation d'un homme en bête à manger, induit systématiquement l'idée d'incorporation qui est le but ultime de cette guerre : incorporer l'autre pour en faire du même. Dans le même temps, les paysans se transforment en résistants, nous pourrions même dire en guerriers. En effet, les deux métamorphoses, celles du Prussien et des paysans, sont solidaires l'une de l'autre parce que dialectiquement unies. La grammaire rituelle sert ici la syntaxe guerrière, d'une part parce le rite du gavage devient un acte de résistance des villageois et d'autre part, parce que l'entente générale autour du rite lève en sourdine l'interdit du meurtre et l'encourage dans un but de légitime défense.

On le voit, la violence guerrière se trace ici un chemin à travers celle du rite, prend sa forme, leurs enjeux se mêlent. Pour reprendre les termes de René Girard dans son chapitre sur " La crise sacrificielle», nous pourrions dire que dans la nouvelle SaintAntoine, «le sacrifice perd son caractère de violence sainte pour se « mélanger» à la violence impure, pour devenir le complice scandaleux de celle-ci ${ }^{(37)} »$ : sang sacrificiel et sang criminel s'épousent. C'est en effet une tactique efficace pour confondre le Prussien. Bataille, pour le citer à nouveau, souligne la violence ambiguë dans laquelle est plongée la victime sacrificielle, « [d]ès qu'elle est consacrée et pendant le temps qui la sépare de la consécration de la mort, elle entre dans l'intimité des sacrifiants $[\ldots]$ : elle est l'un des leurs et, dans la fête où elle périra, elle chante, danse et jouit avec eux de tous les plaisirs. Il n'est plus en elle de servilité ; [...]. Elle est perdue dans l'immense confusion de la fête. Et c'est là justement ce qui la perd. ${ }^{\left({ }^{38)}\right)}$ " C'est un fait que plus les paysans nourrissent le soldat, plus il est affaibli, car il s'attache chaque jour davantage à Antoine, oubliant ainsi son statut de dominant ${ }^{(39)}$.

(37) René Girard, La Violence et le sacré, "Pluriel Philosophie », Hachette Littérature, Paris, 2002 [1972], p. 64.

(38) Georges Bataille, op.cit., p. 64

(39) Cet attachement paradoxal entre le Prussien et Antoine laisse planer l'éventualité d'une union entre les deux hommes. Plusieurs détails dans le texte l'indiquent : ainsi le maire de la commune qui introduit le Prussien chez Antoine est appelé « maître Chicot», nom qui fait sûrement référence à «Chicot Ier, Roi de Carnaval sous Henri III et Henri IV» (voir l'article de Jean-Marie Privat, «Le retour et ses discours. Une ethnocritique des intersignes ", dans Sciences du texte et analyse de discours. Enjeux d'une interdisciplinarité, sous la dir. de J.-M. Adam et U. Heidmann, Etudes de Lettres, Lausanne, 1-2, 2005, pp. 197-227, note 29) et qui laisse présager une union carnavalesque et charivarique; nous avons souligné plus haut que les deux hommes «s'en allaient tous les 
Mais c'est aussi parce que le Prussien n'a pas conscience du rite qui se déroule autour de et à travers lui, qu'il peut aussi innocemment en interrompre le déroulement (et l'efficacité) par une rebuffade des plus légitimes. Et c'est à ce moment, nous l'avons dit, quand le rite se meut en duel et en crime individuel que la nouvelle bascule dans la violence tragique, lorsque le destin, « qui ne fait qu'un avec la violence ${ }^{(40)}$ », prend le pas sur la coutume. Alors qu'Antoine devait venir à bout des Prussiens, il va certes tuer un Prussien, mais il va surtout laisser, par sa faute ${ }^{(41)}$, assassiner un innocent, qui plus est, un représentant de la Nation et de la loi. Ainsi Antoine exécute-t-il, d'une certaine manière, le projet de son ennemi. Le renversement est complet. Alors qu'il fallait faire du Prussien du même, Antoine devient l'autre, l'ennemi de la Patrie.

jours après-midi, bras dessus bras dessous » (774); Antoine n'hésite pas à «pinc[er] les cuisses » (ibid.) de l'Allemand et à «lui tap[er] sur le derrière » (ibid.); les gens du village parle de leur amitié comme d'une «liaison» (779). Nous croyons cependant que la mise en scène avortée de ce couple deux fois transgressif dit de manière carnavalesque et parce que carnavalesque l'impossible alliance entre la France et l'Allemagne.

(40) René Girard, op. cit., p. 75.

(41) «Comme on connaissait leur liaison, on ne le soupçonna pas; et il dirigea même les recherches en affirmant que le Prussien allait chaque soir courir le cotillon. / Un vieux gendarme en retraite, qui tenait une auberge dans le village voisin et qui avait une jolie fille, fut arrêté et fusillé. » (779). 